\title{
HISTORIA ESCOLAR EN CONTEXTO INTERÉTNICO E INTERCULTURAL MAPUCHE*
}

OMAr Turra $^{1}$

\begin{abstract}
RESUMEN: El artículo presenta resultados de una investigación que tuvo como propósito indagar, en las significaciones, respecto de la historia escolar y su enseńanza, que portan estudiantes Mapuches pertenecientes a las identidades territoriales Mapuche-Lafkenche (Tirúa) y Mapuchepehuenche (Alto Biobío), en el contexto de su formación en el sistema escolar chileno. El estudio se realizó desde la metodología cualitativa con un diseño de estudio de casos, utilizándose como técnica de recogida de información el grupo de discusión. Los resultados evidencian una escasa valoración del sector de aprendizaje, débil significatividad de sus propuestas temáticas y presencia de conflictividad y discriminación en su enseñanza.
\end{abstract}

Palabras clave: Historia escolar. Pueblos originarios. Estudiantes Mapuche. Contexto interétnico e intercultural.

\section{SCHOOL HISTORY IN INTERETHNIC AND INTERCULTURAL CONTEXTS OF THE MAPUCHE}

ABSTRACT: The article presents results of a study that aimed at inquiring into the meanings regarding school history and teaching, including students belonging to the Mapuche territorial identities Mapuche-Lafkenche (Tirúa) and Mapuche-pehuenche (Alto Biobío) in the context of their training in the Chilean school system. The study was conducted using a qualitative methodology with a design case study, by means of a group discussion technique for collecting information. The results show a lack of appreciation of the learning sector, weak significance of proposals thematic, and the presence of conflict and discrimination in their teaching.

Keywords: School history. Original populations. Mapuche students. Interethnic and intercultural context.

\footnotetext{
*El artículo se enmarca en el proyecto Fondecyt Regular 1151233: "La historia escolar en contextos interétnicos e interculturales: un estudio desde el sistema escolar en contexto Mapuche/no Mapuche”. ${ }^{1}$ Universidad del Bío-Bío, Región del Bío-Bío - Concepción, Chile. E-mail: oturra@ubiobio.cl DOI: 10.1590/ES0101-73302016140175
} 


\section{HISTOIRE SCOLAIRE EN CONTEXTE INTERETHNIQUE ET INTERCULTUREL MAPUCHE}

RESUMÉ: L'article présente résultats d'une recherche concernant les significations de l'histoire scolaire et son enseignement qu'ont les étudiants mapuche appartenant aux identités territoriales mapuchelafkenche (Tirúa) et mapuche-pehuenche (Alto Biobío) dans le contexte de leur formation scolaire au système chilien. L'étude a été mené à partir d'une méthodologie qualitative, en se basant sur une étude de cas, et utilisant comme technique de collecte de données le groupe de discussion. Les résultats mettent en évidence le peu de mise en valeur du secteur d'enseignement, une faible signification de ses propositions thématiques et la présence d'une conflictivité et d'une discrimination dans l'enseignement.

Mots-clés: Histoire scolaire. Peuples originaires. Etudiants Mapuche. Contexte interethnique et interculturel.

\section{Introducción}

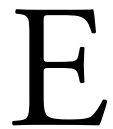

n perspectiva del currículo escolar chileno, el estudiantado perteneciente a un pueblo originario tiene la posibilidad de acceder al conocimiento del pasado histórico-cultural de su pueblo por medio de la asignatura de Historia, Geografía y Ciencias Sociales. A este sector de aprendizaje se atribuye el desarrollo de procesos formativos orientados a potenciar los valores de convivencia y de afirmación identitaria, en el sentido de promover una actitud de respeto a la diversidad histórico-cultural de la humanidad desde la valoración de su propia identidad (MINEDUC, 2009). Definiciones curriculares recientes plantean como el objetivo central de la asignatura el contribuir a que los estudiantes adquieran un sentido de identidad y de pertenencia cultural;

saber quién es, conocer su comunidad y consolidar los lazos con ella son elementos fundamentales para el desarrollo integral de un niño. Ahí radica la base que permite a los alumnos comprender su cultura, apropiarse de ella y participar en su construcción. (MINEDUC, 2013, p. 30).

No obstante, la investigación educativa ha planteado que la historia como asignatura escolar se instituye como uno de los principales dispositivos simbólicos utilizados por grupos hegemónicos, para desplegar procesos formativos orientados a la construcción de identidades nacionales (CARRETERO; CASTORINA, 2010; LÓPEZ FACAL, 2010; PRATS, 2000) y para negar, distorsionar 
y caricaturizar las formas culturales de los pueblos originarios (AMAN, 2010). Solo recientemente, con el reconocimiento de la multiculturalidad de las sociedades y valoración de la diversidad cultural, se ha resignificado la enseńanza de la historia como un ámbito propicio para la conformación de subjetividades interculturales, pues prepara al estudiantado para comprender y reconocer a los otros culturales aportando con ello a la tarea de eliminar males sociales, tales como prejuicios, estereotipos, etnocentrismo y racismo.

Por su parte, los actuales propósitos de la historia escolar se encuentran en consonancia con el reconocimiento de los derechos educativos de los pueblos originarios en Chile, para quienes se ha oficializado el derecho a una formación en su cultura e historia a través del sistema educativo (LEY INDÍGENA, 1993; OIT, 2003).

En alguna medida, la escuela, los docentes, los estudiantes y los demás actores educativos están siendo receptores de estos discursos y cambios curriculares que vienen a tensionar los sentidos y las comprensiones tradicionales de lo que significa enseñar y aprender historia en contextos interétnicos e interculturales, manifestaciones que requieren ser dimensionadas o relevadas. Para el caso del contexto mapuche implica, además, ingresar a un espacio de reconocida conflictividad interétnica y enfrentamiento discursivo que se expresa también en el espacio escolar. Es por ello que en el presente artículo presentamos resultados de una investigación, que tuvo como objetivo indagar en las significaciones respecto de la historia escolar y su enseñanza, que portan estudiantes Mapuches pertenecientes a las identidades territoriales Mapuche-Lafkenche (Tirúa) y Mapuche-Pehuenche (Alto Biobío), en su asistencia y formación en el sistema escolar chileno.

\section{Marco teórico}

Un rasgo distintivo de las sociedades actuales es su carácter multiétnico y multicultural, dada la coexistencia permanente en un espacio territorial de diferentes grupos étnicos con sus repertorios culturales de referencia, que dan origen a dos tipos de relaciones: las interétnicas y las interculturales. Ambas son tipos de relaciones con el otro en que intervienen experiencias, representaciones y códigos valóricos, aunque dan cuenta de realidades específicas y diferenciadas (QUILAQUEO; QUINTRIQUEO; CÁRDENAS, 2005). Las relaciones interétnicas denomina a las relaciones entre grupos que se diferencian y reconocen en su identidad étnica, en sus sistemas simbólicos como la lengua y la religión, entre otras; las interculturales involucran relaciones y encuentros entre grupos humanos diferentes, en donde se entrecruzan los sistemas simbólicos y generan transformaciones culturales entre los grupos que se relacionan (LOSLIER, 1997). Desde esta perspectiva, la educación, la escuela y el currículo conforman espacios simbólicos potencialmente generadores de relaciones interculturales, pero también de posicionamientos étnicos. 
Desde la investigación en la enseñanza de la historia, solo recientemente la alteridad constituye una fuente de preocupación y se justifica a propósito del reconocimiento de la multiculturalidad y valoración de la diversidad cultural por las institucionalidades internacional y estatal. Para el caso europeo, la presencia de estudiantes de ascendencia étnica e inmigrantes en las aulas escolares propone un desafío valórico a la historia escolar, en la medida que su enseñanza favorece la comunicación y tolerancia y ayuda a comprender las diferencias "para poder vivir juntos" (PRATS; BARCA; LÓPEZ FACAL, 2013), pero para ello se requiere relevar funciones educativas específicas de la enseñanza de la historia escolar, en perspectiva multicultural (GALINDO, 2005; VALLS, 2005). Un estudio reciente propone la necesidad de considerar en la enseñanza de la historia una variable de orden cultural que permita al estudiantado inmigrante acceder a las propias historias de sus comunidades de donde provienen, cuestión que implicaría una transformación en las prácticas docentes y un mejoramiento de la formación (GARTH MCCULLOUGH; FRY, 2013). Desde la experiencia en escuelas interculturales chipriotas, se introduce la consideración de la alteridad como una necesidad de época, lo que implica que la enseñanza de la historia debiese consagrar en su trayectoria formativa, o sea la adquisición de las tradiciones culturales de otros pueblos (MAALOUF, 2009).

Para el caso de Estados Unidos, investigaciones en historia escolar también han evidenciado la escasa valoración de la cultura del estudiante inmigrante como saber escolar, y proponen la incorporación de lengua y cultura de referencia como un mecanismo relevante a considerar en su enseñanza y aprendizaje (GIOUROUKAKIS; HONIGSFELD, 2010; MOLL, 1988; OLMEDO, 2003; VERDUGO; FLORES, 2007).

En América Latina, las nociones de multiculturalidad e interculturalidad llegan al ámbito educativo a propósito de las demandas de los pueblos originarios por una educación escolar con pertinencia cultural, puesto que los sistemas educativos excluyeron sus conocimientos socioculturales y educativos del currículo escolar. La respuesta educativa estatal ha otorgado carácter oficial a la educación intercultural bilingüe, reduciéndola a un programa lingüístico-cultural dirigido especialmente a los pueblos originarios, sin comprometer los saberes culturales occidentales del currículo escolar ni tampoco las relaciones interétnicas asimétricas (GALINDO, 2011; QUILAQUEO, TORRES, 2013; QUINTRIQUEO; QUILAQUEO; TORRES, 2014).

En este contexto, la historia escolar si bien ha incorporado el reconocimiento y la valoración de la diversidad cultural en sus orientaciones curriculares, no ha sido mayormente interpelada desde perspectivas interculturales críticas. Sin embargo, es de destacar el trabajo y la propuesta de Ledesma (2006; 2008; 2009), quien sostiene la necesidad de renunciar a viejos y consolidados concep- 
tos, nociones, periodizaciones, universalismos y nacionalismos, para dar espacio a la construcción de una historia escolar abierta a las diversas voces y a los saberes educativos que hasta ahora imposibilita el discurso pedagógico de la historia. Se establece que la diversidad no debe quedarse en lo discursivo en la enseñanza de la historia sino que debe constituirse en una categoría de enriquecimiento cultural, en la medida que permite establecer relaciones con otras formas de concebir el pasado, las nociones de tiempo y las concepciones del mundo (RODRÍGUEZ LEDESMA, 2006). En coherencia con esta línea de argumentación, se encuentra la vertiente de estudios que rescata y releva la memoria histórico-social de las sociedades originarias, la que puede entenderse como un patrimonio cultural o herencia mental, como recuerdos que nutren representaciones, y cohesionan a los individuos en torno a significaciones comunes (LEDUC; MARCOS-ÁLVAREZ; LE PELLEC, 1994). La memoria es cual archivo en la sociedad occidental y se ubica interna y externamente al individuo/colectivo (IGGERS, 2012). En las sociedades originarias, se expresa externamente en lugares ceremoniales y nombres de los lugares vinculados a acontecimientos socio-históricos relevantes y en el patrimonio mental que permite recrear los relatos de la significación histórico-cultural.

En América Latina, la historia escolar se presenta como uno de los principales dispositivos simbólicos utilizados por los grupos culturales hegemónicos para desplegar procesos formativos orientados a la construcción de identidades nacionales y deslegitimar las concepciones de mundo y saberes sociales propios de los pueblos originarios. Desde la época fundacional del Estado y durante buena parte del siglo XX, el discurso pedagógico de la historia como disciplina escolar se asoció a un repertorio de imágenes heroicas que recreaban la trayectoria patria y a una representación del pasado social como constitutiva de una "comunidad cultural", que tenía como propósito explícito interiorizar en las cohortes estudiantiles el sentido de pertenencia a una nación y cultura comunes. La narrativa histórica gestionada ideológicamente por la cultura dominante buscaba unir las conciencias en torno a una causa superior que estaba por encima de cualquier grupo cultural, quedando éstos subsumidos en un marco estatal supra-cultural.

Los nuevos discursos educativos y sociales de reconocimiento de la alteridad indígena, y la promoción de sus derechos educativos y culturales, generan desconcierto en la escuela y sus agentes, si nos atenemos a su sentido histórico-tradicional, una institución pensada para uniformar al alumnado y asimilar culturalmente al indígena. $\mathrm{Al}$ respecto, estudios empíricos realizados desde el ámbito cultural Mapuche plantean la desconsideración del conocimiento indígena en procesos formativos que se desarrollan en las escuelas ubicadas en este contexto (QUILAQUEO; QUINTRIQUEO, 2005) y la prevalencia de un currículo monocultural (QUINTRIQUEO, 2010). Respecto de las actuaciones docentes, se ha evidenciado que el profesorado en sus procesos pedagógicos desarrolla una serie 
de prácticas poco proclives a la consideración del indígena y, más aún, se desarrollan prejuicios y sesgos hacia sus prácticas culturales (QUILAQUEO; MERINO, 2003; UNESCO, 2005).

Desde la perspectiva de la enseñanza de la historia, una investigación que analiza los programas de estudio de la asignatura constató el reducido espacio curricular para la enseñanza de las formas culturales Mapuches, además de la ausencia de una narrativa histórica que otorgue espacio a las visiones de los pueblos originarios, y cuando lo incorpora, lo hace desde una visión del "otro", prevaleciendo una construcción y difusión de una historia nacional hegemónica con un evidente posicionamiento étnico monocultural (TURRA, 2012).

\section{Metodología}

El estudio se realizó en base al paradigma cualitativo de investigación, en tanto sus principios y métodos se orientaban a la búsqueda de alcanzar comprensión respecto de una realidad social determinada, aportada por las significaciones de los propios sujetos que participan del campo objeto de investigación (RODRÍGUEZ; GIL; GARCÍA, 1999).

El enfoque utilizado fue el fenomenológico, puesto que se propuso construir conocimiento a partir de la experiencia subjetiva de los sujetos, describiendo e interpretando los significados comunes aportados por los participantes investigados.

En términos del diseño, se trabajó con la modalidad estudio de casos. Este tipo de estudio permite investigar con cierta intensidad y profundidad un fenómeno social, una institución, un grupo social u otro. Para la investigación, se construyeron dos casos que comprenden a escolares Mapuches representativos de dos identidades territoriales de este pueblo originario. El primero es la identidad Mapuche-Pehuenche, situada en los valles occidentales de la Cordillera de los Andes, que desarrolla su escolaridad en el Liceo Técnico Intercultural Bilingüe de Ralco, comuna de Alto Bío-Bío, donde el porcentaje de etnicidad alcanza, históricamente, a un $85 \%$ de la matrícula de esta institución educativa. El segundo es la identidad Mapuche-Lafkenche, caracterizada por desenvolverse territorialmente en el borde costero de la macro zona Sur, conformada por estudiantes que asisten al Liceo Trapaqueante de la comuna de Tirúa, cuyo porcentaje de etnicidad promedia un 60 de la matrícula.

De acuerdo con los objetivos de la investigación, los casos se construyeron a partir de los siguientes criterios: estudiantes de ascendencia Mapuche, que se encontraban cursando el segundo y tercer medio, y voluntariedad en su participación en el estudio. Basándose en esas variables, se construyeron dos grupos de discusión conformados por ocho escolares cada uno (Tablas 1 y 2). 
Tabla 1

Caracterización del caso "Liceo de Ralco".

\begin{tabular}{l|l|l}
\hline Sujeto & Género & Nivel \\
\hline Estudiante 1 & Femenino & $3^{\circ}$ Medio \\
\hline Estudiante 2 & Masculino & $2^{\circ}$ Medio \\
\hline Estudiante 3 & Masculino & $3^{\circ}$ Medio \\
\hline Estudiante 4 & Masculino & $3^{\circ}$ Medio \\
\hline Estudiante 5 & Masculino & $3^{\circ}$ Medio \\
\hline Estudiante 6 & Femenino & $2^{\circ}$ Medio \\
\hline Estudiante 7 & Femenino & $2^{\circ}$ Medio \\
\hline Estudiante 8 & Femenino & $2^{\circ}$ Medio \\
\hline
\end{tabular}

Tabla 2

Caracterización del caso "Liceo Tirúa".

\begin{tabular}{l|l|l}
\hline Sujeto & Género & Nivel \\
\hline Estudiante 1 & Femenino & $2^{\circ}$ Medio \\
\hline Estudiante 2 & Masculino & $3^{\circ}$ Medio \\
\hline Estudiante 3 & Femenino & $3^{\circ}$ Medio \\
\hline Estudiante 4 & Femenino & $3^{\circ}$ Medio \\
\hline Estudiante 5 & Femenino & $2^{\circ}$ Medio \\
\hline Estudiante 6 & Masculino & $3^{\circ}$ Medio \\
\hline Estudiante 7 & Femenino & $2^{\circ}$ Medio \\
\hline Estudiante 8 & Masculino & $2^{\circ}$ Medio \\
\hline
\end{tabular}

La técnica de recogida de información que se utilizó fue el grupo de discusión, en tanto estrategia que permite confrontar la subjetividad individual con la grupal, y pretende poner en contacto diferentes perspectivas, experiencias y puntos de vista (VALLES, 2007). En tres momentos distintos, se produjo un encuentro entre el investigador y los participantes del caso para alcanzar los propósitos del estudio.

La reducción de la información se realizó de acuerdo al método fenomenológico, codificándose los registros comunicativos que expresan sentidos compartidos o de consenso, es decir la esencia del significado que el estudiantado comparte intersubjetivamente respecto del fenómeno investigado. La concentración de significados comunes muestran las categorías o las unidades del análisis y de ellas se presentan los relatos más representativos como sus resultados. 


\section{Resultados}

Una síntesis de los resultados obtenidos del análisis de la información se expone en la Tabla 3.

Tabla 3

Síntesis de los resultados.

\begin{tabular}{l|c|c}
\hline Categoría & Concentración de significado & $\begin{array}{c}\text { Registros } \\
\text { comunicativos }\end{array}$ \\
\hline Valoración de la clase de historia & Escasa valoración & 6 \\
\hline Problemas históricos de interés & $\begin{array}{c}\text { Colonización, mestizaje, } \\
\text { defensa del territorio }\end{array}$ & 5 \\
\hline $\begin{array}{l}\text { Saberes educativos Mapuche } \\
\text { en la historia escolar }\end{array}$ & $\begin{array}{c}\text { Descontextualización, valoración de los } \\
\text { relatos de abuelos }\end{array}$ & 7 \\
\hline Discriminación en la clase de historia & $\begin{array}{c}\text { Presencia de estereotipos, } \\
\text { cuestionamiento al concepto de indígena }\end{array}$ & 5 \\
\hline
\end{tabular}

\section{Escasa valoración de la clase de historia}

La concentración de sentido se produjo en torno a una escasa valoración por parte del estudiantado respecto de la clase de historia, según se desprende de sus relatos, en tanto se presenta a la asignatura como una instancia en el que se estudian temáticas ocurriendo en tiempos remotos, vinculadas a otras culturas y otros espacios geográficos, y las problemáticas relacionadas con las realidades culturales contextuales no son parte de la clase:

Porque a nosotros nos enseñan historia universal y de repente no entendemos nada, eso que pasó allá, nosotros queremos saber que pasó acá, incluso en nuestra tierra [...] de acá sigue haciendo [...] pasó hace algunos años y a mí me gustaría saberlos, todos los ańos va haciendo historia. - Estudiante 5, Ralco.

Es que en la clase, por mi parte no tocamos ese tema, no hablamos de los Mapuches, hablamos de otro tema, de ¿cómo se llama? [...] Feudalismo, y todas esas cosas. - Estudiante 2, Ralco.

Los recursos didácticos utilizados en su enseñanza resultan poco atractivo al estudiantado:

[...] porque si un profesor explica la materia, uno no se aburre, pero si va y se sienta y dice 'ya desarrollen esta guía' igual es fome, va en la forma del profesor yo creo [...]. - Estudiante 5, Tirúa. 
Es que la profesora no es de esas (que explique la materia), solamente pasa las guerras, se basa en el libro. - Estudiante 7, Tirúa.

Cuando se les pregunta respecto del conjunto de asignaturas que cursan o han cursado y en qué lugar situarían la clase de historia en términos de su interés, la respuesta la ubica entre las menos atractivas: "Las asignaturas que más nos gustan son Biología, Educación Física, Inglés” - varias voces.

En rigor, la escasa valoración que aduce el estudiantado Mapuche no estaría distante de lo que pudiese manifestar un/a estudiante chileno/a que asiste a las clases de historia de un liceo chileno, aunque para el caso se incorpora una situación de impertinencia curricular, en tanto no se consideran saberes educativos propios de la cultura originaria.

\section{Problemas históricos de interés}

La concentración de significado se produjo en torno a la identificación de aprendizajes relevantes con procesos históricos vinculados a la cultura de referencia, o más bien con procesos sociales que vinieron a pauperizar a las culturas originarias. En este sentido, los aprendizajes enunciados como relevantes por los y las estudiantes se pueden definir en las siguientes temáticas: la colonización espańola-chilena; el mestizaje y los contenidos asociados a la defensa del territorio ancestral por los pueblos originarios.

A mí me llama la atención la colonización. Si la colonización chilena [...] en estos territorios. Porque hubieron mucho, este, por ejemplo los españoles se aprovechaban mucho de la gente indígena, igual hubieron hartas muertes, igual por inequidad [...]. Me gustó un poco porque nosotros defendimos nuestro territorio, pero igual no me gustó porque se aprovechaban mucho de las personas. - Estudiante 4, Ralco.

Por mi parte fue cuando Chile hizo una mezcla de todo tipo de personas, entre los blancos y entre los indígenas, y a mí me gustó demasiado porque vivo en un país que está mestizado [...] que yo me acuerde no sé cómo se llamaba cierta persona, por ejemplo la gente zamba, era la gente indígena con negro, entonces yo no sabía que se llamaba así. - Estudiante 1, Ralco.

Para mí las materias de la época de la colonia, donde muestran la vida de los Mapuches, eran interesantes [...]. - Estudiante 7, Tirúa.

Los relatos del alumnado se encuentran en concordancia con los principios del diseńo curricular vigente, lo cual postula el aprendizaje significativo de los/las aprendices y que este comienza con el rescate de las ideas y experiencias 
previas. Además, un aprendizaje será relevante en la medida en que se vincule con las realidades propias de los entornos social y cultural en que se desenvuelven el estudiantado. Al parecer en la clase de historia, no se realizan las debidas vinculaciones temáticas con la realidad del estudiantado Mapuche:

Hacen otros temas pero igual cuando pasan esa materia es interesante. - Estudiante 3, Ralco.

[...] historia Mapuche no vemos mucho, sólo en la básica, en la media no. - Estudiante 4, Ralco.

[...] el problema es que la forma en que está relatada la historia no es concurrente con lo que ha pasado, según mi perspectiva, no es igual. Porque en el libro están relatadas de una manera y en la vida es de otra manera. - Estudiante 8, Tirúa.

La categorización de los aprendizajes relevantes relevados por el estudiantado indígena lleva implícito la demanda por una enseñanza de la historia vinculada a la realidad contextual.

\section{Saberes educativos Mapuches en la historia escolar}

Esta categoría surge desde la indagación en las significaciones del estudiantado Mapuche respecto de la vinculación del saber histórico escolar con su cultura de referencia. Las significaciones en este sentido se concentran, por una parte, en un diagnóstico negativo sobre los saberes y temas que se enseñan en la asignatura:

Nosotros ahora estamos pasando eso que se llama feudalización, feudalismo, y los viajes de Colón, pero todo es como qué países conquistaron, nada de acá, sólo nos pasaron eso. - Estudiante 1, Ralco.

Aquí en historia, por ejemplo, no se toma en cuenta lo que es de la cultura Mapuche, solamente de otras cosas nomás. - Estudiante 6 , Tirúa.

[...] Es que yo creo que la profesora no es muy apegada a la cultura Mapuche, pasa lo que tiene que pasar no más. - Estudiante 4, Tirúa.

Por otra parte, se demanda por una clase más vinculada a la realidad contextual o cultura de referencia del estudiantado:

Yo creo que del $100 \%$ se ve el $45 \%$. Uno viene de la cultura Mapuche, uno tiene ceremonias que se hacen, se siguen haciendo, nosotros queremos eso, estamos conservando y dependiendo más de ello. - Estudiante 2, Ralco. 
[...] no sé hablar, presentarme, decir de dónde procedo, casi lo básico, por eso hay un caballero que nos enseña las historias de antes, lo que sucedía antes, sobre eso se basa y nos enseña, es un asesor cultural. - Estudiante 8, Tirúa.

El alumnado expone como alternativa a un conocimiento histórico descontextualizado, el diálogo con los abuelos, es decir, la recurrencia a la tradición oral, fuente legítima de la transmisión de conocimiento en las culturas originarias:

[...] de repente le pregunto a mis abuelos por qué la gente vive tan aislada, no vive en la ciudad, y me dicen que hay un problema con la gente, con los españoles y todo eso. - Estudiante 1, Ralco.

[...] no nos hablaban de historia; los abuelitos nomás, es interesante lo que cuentan ellos, porque está más relacionado con la vida que lo otro. - Estudiante 5, Ralco.

Vale resaltar en estas observaciones, realizadas por los estudiantes, que los abuelos son considerados aún por ellos como fuentes de conocimiento legítimo para adquirir aprendizajes respecto del pasado histórico-cultural de su pueblo originario.

Sin embargo, al discutir la posibilidad de incorporar temas vinculados a su realidad cultural en la clase, muchos de ellos estuvieron en desacuerdo con aquello, pues sería una fuente de conflictos con escasos compañeros hijos de colonos chilenos con los que comparten clases.

Porque cuando hablan de temas entre Mapuches y españoles, yo veo a mi compañero del lado que no es Mapuche, entonces me siento como [...] porque me siento con él, me entra un rencor. A mí me pasó eso cuando estaba en primero, y me juntaba con compañeros que no eran Mapuches, entonces cuando hablaban de esos temas, me sentía, así, como enejada con sus descendientes. - Estudiante 2, Ralco.

Yo estoy de acuerdo con las respuestas de mis compañeros, porque tenemos muchos conflictos con los compañeros y profesores también porque a veces hay una mala comprensión de ciertas cosas. - Estudiante 4, Ralco.

Es que en nuestro colegio no hay alumno que sea tan apegado a su descendencia, yo creo que va en eso, no hay en nuestro curso alguien que le guste harto su cultura, porque si lo hubiera, pelearíamos, defenderíamos nuestra cultura y no aceptaríamos todo lo que nos pasan [...]. - Estudiante 7, Tirúa.

Las observaciones manifestadas por los alumnos Mapuches es que a pesar de ser ellos mayoría en sus respectivos establecimientos y en la clase, perciben 
que los temas relacionados con su cultura son fuente de discusión y conflicto al profesorado y para el escaso número de compañeros/as chilenos/as que componen sus cursos; prefieren por lo mismo no agregar otros temas a la clase de historia que pudiesen profundizar estos conflictos.

\section{Discriminación en la clase de historia}

Una categoría que emergió de la discusión grupal de manera transversal a las preguntas planteadas, sin asociación explícita con ninguno de los objetivos planteados, fue aquella vinculada a las declaraciones de discriminación en la clase de historia. Los y las estudiantes Mapuches manifestaron que en la clase existían expresiones y acciones del profesorado y de sus compañeros/as chilenos/as que ofenden, o al menos les generaba desagrado:

Una vez teníamos un profesor que escribió en la pizarra, los Pehuenche son unos ignorantes, borrachos y otras cosas más; entonces uno siente rabia. -Estudiante 4, Ralco.

Hay un tema de discriminación, porque para hablar de los temas, se tiene que ver más la cosa, yo soy hablante de Chedungun, hay compañeros (chilenos) que si uno habla se empiezan a llevar mal, yo como indígena esas cosas que pasan en historia, como por ejemplo, ya mataron a un indígena, a mí me empieza a dar rabia una cosa así, creo que hay muchas contradicciones entre chilenos y nosotros, la clase de historia para mí sería mejor, yo la dejaría de lado, porque empiezan como a discriminarse y como ahora estamos todos juntos comienzan a generarse conflictos, porque siguen recordando siempre lo mismo, por eso [...]. - Estudiante 2, Ralco.

[...] A los profesores no les interesa si hay más estudiantes Mapuches, pasan las materias del libro y los libros los manda el Gobierno y yo creo que el Gobierno es más discriminatorio. Estudiante 6, Tirúa.

Muchas veces, la enseñanza de la historia por sus problemáticas es fuente de conflictos y discusiones, y también es deseable que generen conflicto en el estudiantado - sobre todo si es de carácter cognitivo —-, pero obviamente sin que ello genere afectaciones o discriminaciones que afecten a individuos o grupos dentro de la clase.

A mí no me gusta hablar mucho de este tema porque a mí mismo me da mucha rabia que me digan indígena, prefiero que me digan Mapuche o Pehuenche, pero indígena, lo escuchamos mucho en la clase y uno tiene que guardarse la opinión. - Estudiante 3, Ralco. 
La citada demanda del estudiante no es menor, considerando la impronta o carga histórica negativa que lleva el concepto de indígena, y las declaraciones curriculares, e incluso las legales (Ley Indígena), la incorporan como lenguaje aceptado, pero a los sujetos alejados/as de estos centros de configuración conceptual les incomoda, o definitivamente las rechazan.

\section{Discusión}

Un primer aspecto a discutir, refiere a que los nuevos propósitos educativos de la enseńanza de la historia vinculados a la afirmación identitaria y la promoción de valores de convivencia intercultural, de acuerdo con los hallazgos investigativos, no se han manifestado en la experiencia educativa de los escolares Mapuches. Si bien la historia escolar ha incorporado el reconocimiento y la valoración de la diversidad cultural en sus orientaciones curriculares, ello no se ha traducido en la implementación de procesos pedagógicos que integren saberes educativos de los pueblos originarios en su enseñanza, persistiendo una organización curricular y un cuerpo de contenidos asociados a la tradición europea occidental y a la trayectoria de la sociedad colonial/criolla/chilena, ancladas en un tradicional discurso pedagógico de la historia (TURRA, 2015; RODRÍGUEZ LEDESMA, 2006). Es decir, prima una perspectiva monocultural hegemónica que guía su organización como disciplina escolar.

Desde esta perspectiva, la enseñanza de la historia imposibilita el ejercicio de un pensamiento histórico que construya y favorezca la diferencia y la alteridad en el alumnado, cuestiones fundamentales si se postula la afirmación identitaria y la construcción de subjetividades interculturales como finalidades de su enseñanza. Por el contrario, basándose en esta comprensión, la historia escolar se configura como una expresión de la colonialidad del poder (QUIJANO, 2000), en tanto asigna superioridad a unos conocimientos derivados del mundo europeo y excluye, omite y silencia aquellos de las culturas originarias, produciendo una sub-alternización de su conocimiento y lenguaje.

Esta persistencia pedagógica-curricular encuentra coherencia con los planteamientos investigativos que constatan que los cambios promovidos en el currículo están sujetos a complejos procesos de significación y reflexión de los profesores, los cuales involucran entre otros aspectos los códigos propios de las disciplinas escolares y los conocimientos profesionales que poseen los docentes (FULLAN, 2010; HARGREAVES et al., 2001; GOODSON, 1995). Esos aspectos contribuyen al fortalecimiento de la cultura escolar en la que el profesorado representa la legitimidad socioprofesional de los campos disciplinarios que enseña, excluyendo otros saberes y agentes educativos.

Los relatos del estudiantado originario dan cuenta del potencial interés por temáticas propias de su cultura de referencia y/o lejanía con la 
propuesta temática de la historia escolar, cuestión que implica pensar una historia escolar en perspectiva intercultural que entre en diálogo con otras formas de concebir el pasado, de organizar el conocimiento y de estar en el mundo, es decir una disciplina escolar capaz de dar espacio a la heterogeneidad histórico-estructural y la construcción de una inter-episteme que integre otros principios de conocer y entender. Aquello involucra superar la colonialidad del ser y saber cómo un proceso por el cual se construyó y se consolidó una forma de pensamiento hegemónico - eurocéntrico, lineal y totalizante - que se universalizó en las sociedades coloniales y que se encarnó en las disciplinas escolares (LANDER, 2000; MIGNOLO, 2003; CASTRO-GÓMEZ; GROSFOGUEL, 2007).

Un segundo ámbito es la manifestación de situaciones de conflictividad y discriminación en la clase de historia, expresiones que sin duda se inscriben en el proceso histórico de construcción del social y cultural en el contexto Mapuche/no Mapuche, en el que la dialéctica de configuración de identidades se ha desarrollado en el marco de la conflictiva relación del estado chileno con el pueblo Mapuche, o para ser más preciso, en un contexto de relaciones interétnicas violentas y asimétricas promovidas desde el estado nacional. En este proceso, la escuela ha jugado un papel central en la construcción de comunidades imaginadas, en tanto productora y transmisora cultural de la idea de nación (ANDERSON, 1993), pues a través de ella el Estado consolidó y/o consolida su hegemonía cultural en la medida que controla los mecanismos de producción simbólica y de distribución del conocimiento (ROJAS; CASTILLO, 2005). En este sentido, los discursos que circulan en la escuela son formaciones discursivas más amplias (políticas, económicas, sociales, culturales etc.), que han ido configurando el conocimiento escolar oficial, las identidades y las posiciones que ocupan los diversos sujetos en los espacios de relaciones sociales.

Por lo anterior, las expresiones de conflictividad y discriminación que exponen los escolares Mapuches refiéranse a fenómenos propios de sociedades en contacto de relaciones interétnicas asimétricas, en el que se manifiestan situaciones de dominación y control simbólico, que para el caso, una mayoría cultural en el contexto Mapuche/no Mapuche y estudiantil en el centro educativo $(85$ y $60 \%$ ) resulta minorizada culturalmente, al desplegarse unos objetivos y unas finalidades educativas que se corresponden con dimensiones propias de la sociedad y cultura dominante. Las características socioculturales del alumnado participante del estudio, que en su mayoría conserva pautas culturales tradicionales en la organización de su existencia, su situación sociolingüística que hace de ellos/as hablantes de la lengua originaria (mapuzugun), expresan una identidad cultural de base que contribuye a explicar las situaciones de conflictividad generadas en la enseñanza de la asignatura y la discriminación percibida en relaciones pedagógicas. 
Por último, los resultados del estudio vienen a confirmar los hallazgos investigativos que constatan, para contextos específicos de indigeneidad Mapuche, la exclusión de los saberes culturales originarios en programas de estudio (QUILAQUEO; QUINTRIQUEO; CÁRDENAS, 2005; QUINTRIQUEO, 2010) y la existencia de situaciones de prejuicios y discriminación hacia las formas culturales indígenas por parte del profesorado (QUILAQUEO; MERINO, 2003; UNES$\mathrm{CO}, 2005)$. También se constata, desde la perspectiva del estudiantado Mapuche, la ausencia de una narrativa histórica que otorgue espacio a las visiones de los pueblos originarios (TURRA, 2012; 2015).

Respecto del profesorado, protagonista secundario en este estudio pero sin duda fundamental en los procesos de significación y valoración de la enseñanza de la historia que realiza el estudiantado, sus actuaciones en el contexto de estudio llevan a sospechar que cuando el discurso educativo demanda nuevas prácticas de enseñanza, para responder a los desafíos de la interculturalidad, se impone un requerimiento de competencias profesionales al profesorado para el cual no fue habilitado en la formación inicial, situación que debiese constituirse en un desafío para las instituciones formadoras de profesores.

\section{Conclusiones}

La historia escolar ha incorporado el reconocimiento y la valoración de la diversidad cultural en sus orientaciones curriculares, sin embargo ello no se ha traducido en la adición de saberes histórico-educativos de los pueblos originarios en su enseñanza, persistiendo una organización curricular y unas formas de enseñar ancladas en el tradicional discurso pedagógico de la historia.

En un contexto social interétnico e intercultural, como el estudiado, la historia escolar se representa como un dispositivo cultural privilegiado en la proyección de la colonialidad, en tanto mecanismo de producción simbólica y de distribución del conocimiento, que valida unos contenidos escolares y una perspectiva de estudio de tradición eurocéntrica, además sub-alternaliza, por el contrario, los conocimientos y sentidos culturales de los pueblos originarios.

Las significaciones aportadas por los estudiantes Mapuches sobre la historia escolar - problemas históricos de interés, saberes Mapuches en su enseñanza, conflictividad y discriminación en el aula - dan cuenta de prácticas docentes asociadas a una enseńanza tradicional de la historia que, por cierto, contribuye a una escasa valoración del sector de aprendizaje entre el alumnado.

En consideración a los derechos educativos de los pueblos originarios, al reconocimiento de la multiculturalidad de las sociedades actuales, urge avanzar hacia una historia escolar intercultural que incorpore y dialogue con otras formas de comprender el pasado, organizar el conocimiento y concebir el mundo. 


\section{Referencias}

ANDERSON, B. Comunidades imaginadas: reflexiones sobre el origen y la difusión del nacionalismo. Buenos Aires: Fondo de Cultura Económica, 1993.

AMAN, R. El indígena "latinoamericano" en la enseñanza: representación de la comunidad indígena en manuales escolares europeos y latinoamericanos. Estudios Pedagógicos, v. 36, n. 2, p. 41-50, 2010.

CARRETERO, M.; CASTORINA, J. La construcción del conocimiento histórico. Enseñanza, narración e identidades. Buenos Aires: Paidós, 2010.

CASTRO-GÓMEZ, S.; GROSFOGUEL, R. Prólogo: Giro decolonial, teoría crítica y pensamiento heterárquico. En: CASTRO-GÓMEZ, S.; GROSFOGUEL, R. (Editores). El giro decolonial. Reflexiones para una diversidad epistémica más allá del capitalismo global. Bogotá: Iesco-Pensar-Siglo del Hombre Editores, 2007. p. 09-23.

GALINDO, R. Cambios curriculares en Ciencias Sociales para responder a la multiculturalidad. En: GARCÍA, C.; GÓMEZ, E.; JIMÉNEZ, M.; LÓPEZ, J.; MARTÍNEZ, J.; MORENO, C. (Editores). Enseñar Ciencias Sociales en una sociedad multicultural: una mirada desde el Mediterráneo. Almería: Asociación Universitaria del profesorado de Didáctica de las Ciencias Sociales, 2005. p. 305-343.

FULLAN, M. Investigación sobre el cambio educativo: presente y futuro. Revista Mexicana de Investigación Educativa, v. 15, n. 47, p. 1100-1106, 2010.

GARTH MCCULLOUGH, R.; FRY, M. Every community has a story: The impact of the bilingual history fair on teaching and student learning. The Journal of Social Studies Research, v. 37, n. 3, p. 151-165, 2013.

GIOUROUKAKIS, V.; HONIGSFELD, A. High-stakes testing and English language learners: using culturally and linguistically responsive literacy practices in the high school English classroom. Tesol Journal, v. 1, n. 4, p. 470-499, 2010.

GOODSON, I. Historia del Curriculum. La construcción social de las disciplinas escolares. Barcelona: Pomares, 1995.

HARGREAVES, A.; EARL, L.; MOORE, S.; MANNING, S. Learning to change. Teaching Beyond Subjects and Standards. San Francisco: Jossey-Bass/Wiley, 2001.

IGGERS, G. La historiografía del siglo XX: desde la objetividad científica al desafío posmoderno. Santiago: Fondo de Cultura Económica, 2012.

LANDER, E. La colonialidad del saber: eurocentrismo y ciencias sociales. Perspectivas latinoamericanas. Buenos Aires: Consejo Latinoamericano de Ciencias Sociales, 2000.

LEDUC, J.; MARCOS-ALVAREZ, V.; LE PELLEC, J. Construire i’historie. Toulouse: Bertrand-Lacoste-CRDP Midi Pyrénées, 1994.

LOSLIER, S. Des relations interculturelles: du roman à la réalité. Montreal: Liber, 1997.

LÓPEZ FACAL, R. Nacionalismos y europeísmos en los libros de texto: Identificación e identidad nacional. Clío \& Asociados, n. 14, p. 9-33, 2010. Memoria Académica. Disponible 
en: <http://www.memoria.fahce.unlp.edu.ar/art revistas/pr.4018/pr.4018.pdf >. Acceso en: 25 ago. 2014.

MAALOUF, A. El desajuste del mundo. Madrid: Alianza Editorial, 2009.

MIGNOLO, W. D. Desobediencia epistémica: retórica de la modernidad, lógica de la colonialidad y gramática de la descolonialidad. Buenos Aires: Ediciones del Signo, 2010.

MINISTERIO DE EDUCACIÓN - MINEDUC. Historia, Geografía y Ciencias Sociales. Programa de estudio Sexto Año Básico. Santiago, 2013. Disponible en: <http:// www.curriculumenlineamineduc.cl/605/articles-18973_programa.pdf $>$. Acceso en: 27 ago. 2014 .

- Objetivos fundamentales y contenidos minimos obligatorios de la educación básica y media. Santiago: Unidad de Currículum y Evaluación, 2009.

MOLL, L. C. Some key issues in teaching Latino students. Language Arts, n. 65, p. 465472, 1988.

ORGANIZACIÓN INTERNACIONAL DEL TRABAJO - OIT. Convenio Número 169 sobre Pueblos Indigenas y Tribales: Un Manual. Francia. OIT, 2003. Disponible en: <http://pro169.org/res/materials/es/general_resources/Convenio\%20num\%20169\%20 \%20manual.pdf>. Acceso en: 27 ago. 2014.

OLMEDO, I. M. Accommodation and resistance: Latinas struggle for their children's education. Anthropology and Education Quarterly, v. 34, n. 4, p. 373-395, 2003.

PRATS, J.; BARCA, I.; LÓPEZ FACAL, R. (Eds.). Historia e identidades culturales. Braga: Universidade do Minho, 2013.

PRATS, J. Dificultades para la enseñanza de la historia en la Educación Secundaria: reflexiones ante la situación española. Revista de Teoría y Didáctica de las Ciencias Sociales, n. 5, p. 71-98, 2000.

QUIJANO, A. Colonialidad del poder, eurocentrismo y América Latina. Buenos Aires: Clacso-Unesco, 2000.

QUILAQUEO, D.; MERINO, M. Estereotipos y prejuicio étnico hacia los mapuches en textos complementarios a la asignatura de Historia. Campo Abierto, n. 23, p. 119-135, 2003.

QUILAQUEO, D.; QUINTRIQUEO, S.; CÁRDENAS, P. Educación, Currículum e interculturalidad. Elementos sobre formación de profesores en contexto mapuche. Temuco: Ediciones Universidad Católica de Temuco, 2005.

QUILAQUEO, D.; TORRES, H. Multiculturalidad e interculturalidad: Desafios epistemológicos de la escolarización desarrollada en contextos indígenas. Alpha, n. 37, p. 285-300, 2013.

QUINTRIQUEO, M.; QUILAQUEO, R.; TORRES, H. Contribution for the teaching of natural sciences: Mapuche and school knowledge. Educação e Pesquisa (En proceso de publicación), 2014.

QUINTRIQUEO, S. Implicancias de un modelo curricular monocultural en contexto mapuche. Temuco: Ediciones Universidad Católica de Temuco, 2010. 
RODRÍGUEZ, J.; GIL, J.; GARCÍA, E. Metodología de la investigación cualitativa. Málaga: Aljibe, 1999.

RODRÍGUEZ LEDESMA, X. Historia y diversidad. El lugar de la otredad en la reconversión del paradigma imperante. Cuidado de la edición y diseño: estirpe, concepto e imagen, n. 29, 2009.

Historia y educación en un mundo multicultural. En: Convergencia con Europa y cambio en la universidad: XI Conferencia de Sociología de la Educación: Santander, 22, 23, y 24 de septiembre de 2006, p. 186-187.

- Una historia desde y para la interculturalidad. Buenos Aires: Universidad Pedagógica Nacional, 2008.

ROJAS, A.; CASTILLO, E. Educar a los otros. Estado, políticas educativas y diferencia cultural en Colombia. Serie Estudios Sociales. Colección Cultura y Educación. Popayán: Editorial Universidad del Cauca, 2005.

SANTIAGO. Ley Indígena 19.253. Protección, Fomento y Desarrollo de los Indígenas, y crea la Corporación Nacional de Desarrollo Indígena. Santiago: Biblioteca del Congreso Nacional de Chile, 1993.

TURRA, O. Profesorado y saberes histórico-educativos mapuche en la enseñanza de historia. Revista Electrónica Educare, v. 19, n. 3, p. 1-20, 2015.

. Currículo y construcción de identidad en contextos indígenas chilenos. Educación y Educadores, v. 15, n. 1, p. 81-95, 2012.

UNESCO. La discriminación y el pluralismo cultural en la Escuela. Casos de Brasil, Chile, Colombia, México y Perú. Santiago: OREAL/UNESCO, 2005.

VALLES, M. Técnicas cualitativas de investigación. Madrid: Editorial Síntesis, 2007.

VALLS, R. La multiculturalidad en la enseñanza de la historia y de las ciencias sociales: el desajuste entre intenciones educativas y prácticas escolares y los retos del futuro educativo inmediato. En: GARCÍA, C.; GÓMEZ, E.; JIMÉNEZ, M.; LÓPEZ, J.; MARTÍNEZ, J.; MORENO, C. (Editores). Enseñar Ciencias Sociales en una sociedad multicultural: Una mirada desde el Mediterráneo. Almería: Asociación Universitaria del Profesorado de Didáctica de las Ciencias Sociales, 2005. p. 263-277.

VERDUGO, R.; FLORES, B. English-language learners. Education and Urban Society, v. 39, n. 2, p. 167-193, 2007.

Recibido en 04 de junio de 2014.

Aprobado en 24 de marzo de 2016. 